\title{
Excel como una herramienta asequible en la enseñanza de la Estadística.
}

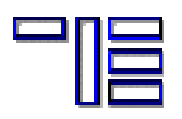

\author{
Myrna López Noriega \\ mdlopezepampano. unacar.mx \\ Cristina Lagunes Huerta \\ clagunesepampano. unacar.mx \\ Santa Herrera Sánchez \\ sherreralpampano.unacar.mx \\ Universidad Autónoma del Carmen. Campeche. \\ México.
}

\section{Introducción}

Actualmente la presencia de la computadora en los programas educativos se ha vuelto una constante. En las últimas décadas en los llamados países desarrollados el coeficiente de estudiante por computadora ha ido cambiando drásticamente, tal es el caso de Estados Unidos donde en Dakota del Norte se informa que este es de dos estudiantes por una computadora[1]. Ciertamente nuestros países aún están lejos de este índice pero, no podemos dejar de reconocer la presencia cada vez mayor de esta herramienta en las instituciones educativas.

De la misma forma en que ha aumentado la disponibilidad de computadores en la educación y en sus programas, también lo ha hecho su uso. En la actualidad, es común que los programas y asignaturas en muchas instituciones de educación Básica y Media incluyan la utilización del procesador de texto, mientras que en el nivel medio superior les soliciten el usar CD ROMS o bien el navegar en la Red.

El Consejo Nacional de Profesores de Matemáticas (NCTM, por sus siglas en inglés) declara que la Tecnología es una herramienta básica para la enseñanza y el aprendizaje efectivos de las matemáticas; amplía las matemáticas que se pueden enseñar y mejoran el aprendizaje de los estudiantes.

Para ello justifican su uso aduciendo que: "Las calculadoras, el software de herramientas del computador, y otras tecnologías ayudan en la recolección, grabación, organización y análisis de datos. Aumentan además la capacidad de hacer cálculos y ofrecen herramientas convenientes, precisas y dinámicas que dibujan, grafican y calculan. Con estas ayudas, los estudiantes pueden extender el rango y la calidad de sus investigaciones matemáticas y enfrentarse a ideas matemáticas en ambientes más realistas.

Las nuevas tecnología han venido a cambiar por completo el panorama tradicional como se hacían, se veían y se enseñaban las matemáticas (Orozco, 2004). Insertarse en este nuevo panorama implica realizar profundo cambios en nuestros programas educativos, reconociendo la mediación que juega la computadora en la relación cognitiva que se establece entre sujeto y objeto del conocimiento.

En el contexto de un programa de matemáticas bien articulado, la tecnología acrecienta tanto el alcance del contenido matemático como el rango de situaciones problemáticas o tipos de problemas al que pueden enfrentarse los estudiantes. Herramientas de computo poderosas, construcciones y representaciones visuales ofrecen a los estudiantes acceso a contenidos matemáticos y a contextos que de otro modo serían para ellos muy difíciles de explorar. El uso de las herramientas tecnológicas para trabajar en contextos de problemas interesantes puede facilitar el logro de los estudiantes en una variedad de categorías de aprendizaje de orden superior tales como reflexión, razonamiento, 
planteamiento de problemas, solución de problemas y toma de decisiones.’[2]

Finalmente confirman la presencia de la tecnología como una herramienta esencial dentro de "un programa matemático balanceado". Para lograr lo anterior los profesores necesitamos de una preparación que nos permita establecer cuándo y cómo se pueden incluir efectiva y coherentemente estas herramientas en los programas educativos.

Si bien, el uso más frecuente de las computadoras por parte de los estudiantes es con el procesador de texto, no podemos desconocer el enorme océano de posibilidades y ventajas que representa para cualquier estudiante el manejo de la hoja de cálculo.

\section{La Hoja de Cálculo como herramienta}

La Hoja de Cálculo puede convertirse en una poderosa herramienta para crear ambientes de aprendizaje que enriquezcan la representación (modelado), comprensión y solución de problemas, especialmente en el área de matemáticas. Desafortunadamente, la mayoría de docentes y estudiantes nos limitamos a utilizar sólo funciones básicas de ella, como tabular información y realizar cálculos mediante formulas, desconociendo que ofrece funcionalidades que van más allá de la tabulación, cálculo de fórmulas y graficación de datos, permitiendo crear y hacer uso de simulaciones que posibilitan a los estudiantes para realizar representaciones que permiten construir un puente entre las ideas intuitivas y los conceptos formales.

Desde sus inicios las Hojas de Cálculo lograron un éxito rotundo debido a que su uso en los problemas cotidianos y reales en el mundo empresarial representaba una oportunidad de llevar esos problemas reales y representarlos a través de números y fórmulas. Pese a lo anterior, la forma de utilizar las TICs en la educación no se ha llevado de igual manera, "donde muy a menudo la instrucción se orienta hacia lo que "podría" hacer la herramienta y no a los problemas que ayuda a resolver".[3].

Nos hemos olvidado de la verdadera esencia de la Hoja de Cálculo que provee magníficos ambientes para el estudio de la representación (modelado) de problemas, para el uso de fórmulas en cálculos matemáticos y para la solución de diversos problemas de variadas disciplinas. La creación de este tipo de ambientes es un reto que debemos asumir los maestros; la hoja de cálculo puede contribuir sustancialmente al mejoramiento de la educación de estudiantes de todas las edades.

La maestra Pamela Lewis[4], autora del libro "La Magia de la Hoja de Cálculo", considera que esta es una herramienta de aprendizaje poderosa y que si los estudiantes tienen acceso a computadores, deben utilizarla. Argumenta que desarrolla en los estudiantes habilidades para:

a. organizar datos (ordenar, categorizar, generalizar, comparar y resaltar los elementos claves);

b. realizar diferentes tipos de gráficas que agreguen significado a la información ayudando en la interpretación y análisis;

c. utilizar gráficas para reforzar el concepto de porcentaje;

d. identificar e interpretar para un conjunto de datos, el máximo y mínimo, media, mediana y moda;

e. utilizar elementos visuales concretos con el fin de explorar conceptos matemáticos abstractos (inteligencia visual y espacial);

f. descubrir patrones;

g. comprender conceptos matemáticas básicos como conteo, adición y sustracción;

h. estimular las capacidades mentales de orden superior mediante el uso de fórmulas para responder a preguntas condicionales del tipo "si... entonces";

i. solucionar problemas y

j. usar fórmulas para manipular números, explorar cómo y qué formulas se pueden utilizar en un problema determinado y cómo cambiar las variables que afectan el resultado. 
De acuerdo a las investigaciones (Hernando, 2003) tenemos que los estudiantes que se enfrentan a diversos temas matemáticos, como funciones, utilizando una Hoja de Cálculo y herramientas gráficas, logran mayor comprensión que quienes lo hacen de manera tradicional . Además, el uso de computadoras ayuda a sostener el interés del estudiante al momento de desarrollar tareas que son tediosas si se llevan a cabo de la manera tradicional, con lápiz y papel.

\section{Excel y la Estadística}

Si nos enfocamos al uso de la hoja de cálculo en la Estadística, es importante primero, realizar algunas consideraciones detectadas a lo largo de varios años de guiar en el conocimiento de esta disciplina, antes de entrar en materia.

En la actualidad se está reconociendo la importancia de la probabilidad y la estadística dentro de los programas educativos, como una asignatura que fortalece la toma de decisiones.

A nivel universitario los cursos de estadística se incluyen prácticamente en todas las especialidades, ya sea como probabilidad y estadística, bioestadística, teoría del muestreo o con algún otro nombre, hoy en día la estadística se encuentra presente en los diferentes campos de la ciencia.

Para empezar a trabajar en la estadística no se necesita un dominio profundo de la matemática, basta con la comprensión de los propios conceptos estadísticos.

La preparación estadística actual incluye la habilidad de procesamiento de datos a través de un programa estadístico.

Esta realidad nos obliga, de acuerdo a nuestros recursos, a acercar a los estudiantes a programas de análisis estadístico, considerando que el rápido desarrollo de la estadística y su difusión en la época moderna se ha debido a la presencia de la computadora facilitando la acelerada cuantificación de nuestra sociedad y al modo en que los datos son recogidos y procesados.[5]

También debemos considerar que como materia del área de las matemáticas, la computadora no es sólo un recurso de cálculo, sino también un recurso didáctico potente y muy útil, que nos permite conseguir una aproximación más exploratoria y significativa en la enseñanza de la estadística[6]. Por sí misma la computadora nos permite lograr ciertos avances en el aprendizaje pues evita: la realización de cálculos tediosos, y pocos constructivos; las largas jornadas desgastantes en la introducción de enormes conjuntos de datos; y facilita la actualidad y posible adecuación de las prácticas a los intereses personales de los estudiantes cuando se conjuga con Internet. Brindando por todo ello, un efecto por demás motivador en el estudiante (Hernando 2003).

Hasta hace algunos años, el análisis de datos estaba reservado a profesionales, quienes tenían que diseñar sus propios programas para realizar los cálculos, poco podíamos esperar de la experiencia educativa. Sin embargo en los últimos veinte años, el campo de la estadística se ha transformado radicalmente gracias al desarrollo de programas de computadora especialmente diseñados para el análisis estadístico.’Durante los ochenta, el software estadístico experimentó una vasta revolución tecnológica. Además de las mejoras manifestadas en actualizaciones periódicas, la disponibilidad de computadoras personales condujo al desarrollo de nuevos paquetes que usaban una interfaz manejada por menús"[7]

Esta revolución tecnológica trajo consigo una gran variedad de programas estadísticos, tanto de tipo profesional, como los desarrollados con fines educativos. Paquetes estadísticos profesionales como SAS, SPSS, SYSTAT, STATISTICS, etc. aparecieron en el mercado informático.

También tenemos los desarrollados especialmente para ser usados en la enseñanza, como MINITAB. Toda esta paquetería representó al final de la década de los ochenta y el principio de los noventa la realidad de un período continuo de avances tecnológicos manifestados en procedimientos estadísticos 
adicionales cada vez más sofisticados.

Es evidente que el uso de los paquetes de software es algo común dentro de los ambientes empresariales, de investigación y académicos. Así, tenemos entornos operativos "amistosos" , que permiten acceder directamente al manejo de cualquiera de los módulos de un paquete estadístico y, con la ayuda del "ratón", explorar sus posibilidades.

Por otro lado, existen programas "de consulta" (Brent y Mirelli, 1991) a los cuales se puede recurrir para obtener un "consejo" sobre el método de análisis que se debe aplicar en función del tipo de datos y las hipótesis sobre los mismos.

Sin embargo, tenemos que reconocer que pese a la variedad de software existentes en el medio, la realidad de la educación en nuestros países, en nuestras universidades supera muchas veces las expectativas de los programas; la situación económica por la que atraviesan la mayoría de las instituciones nos obliga a buscar alternativas económicas y asequibles a nuestros estudiantes, que permitan llevar los conceptos estadísticos y su metodología a la práctica a través de aplicaciones informáticas, logrando con ello manipular formulas y variables de acuerdo a un problema determinado.

Por otro lado, en años de experiencia con alumnos del área administrativa, cuyos conocimientos de matemáticas no son profundos, hemos detectado que para poder trabajar en estadística con estudiantes aún "inexpertos" en programas estadísticos necesitamos que el software utilizado cumpla determinadas condiciones:

Un proceso de instalación automático y sencillo.

Introducción sencilla de la información.

Definición de variables y creación de ficheros de forma intuitiva.

Importación y exportación de datos desde los formatos más habituales

Operadores (aritméticos, lógicos y relacionales).

\section{Funciones}

La hoja de cálculo de Excel, ha cumplido perfectamente con estas condiciones y si tomamos en cuenta que por formar parte del paquete integrado Microsoft Office (en cualquiera de sus versiones) se encuentra prácticamente a la mano de cualquier estudiante, lo que le ha permitido permanecer por su sencillez, disponibilidad y relación calidad/precio como una herramienta idónea tanto para estudiantes, usuarios principiantes como para maestros usuarios avanzados, que deseen realizar una análisis estadístico.

Excel, dependiendo de la versión, es un programa que se ajusta bastante bien al contenido mínimo de muchos de los programas educativos y que cumple con las condiciones señaladas anteriormente (en el caso de Excel 1997 y Excel 2000 carecen de algunas funciones ya actualizadas en el XP). Se trata de un software considerado actualmente como estándar en todos los entornos (educativo, profesional, familiar, etc), que posee la virtud de presentar una interfaz agradable y una facilidad de uso digna de elogio[8].

Cabe señalar algunas de las ventajas que hemos identificado en la práctica, al trabajar procedimientos estadísticos, inclusos aquellos considerados como difíciles.

Las funciones utilizan una sintaxis común de acuerdo al nombre de estas, olvidándose de aquella típica de programación. 
Al utilizar funciones de aplicación inmediata optimizan la funcionalidad. diálogos.

Al aplicar las funciones se realiza la retrolimentación de conceptos a través de sus cuadros de

El programa integrado de Office por sí mismo ofrece una gran ayuda.

Tiene una capacidad gráfica muy variada que permite trabajar en cualquiera de las etapas de una análisis estadístico.

Tiene la posibilidad de automatizar cualquier tarea repetitiva a través del uso de macros de fácil aplicación.

También tenemos que considerar que la instalación del programa mismo es muy sencillo, requiriendo características mínimas muy básicas que se encuentran prácticamente disponibles en cualquier computadora actualmente, y por si fuera poco, el programa no ocupa mucho espacio en el disco duro y por ende no necesita demasiada memoria para funcionar.

Como una bondad adicional, Microsoft Excel incluye un comando para el análisis de datos, dentro de las "herramientas para análisis", que se pueden utilizar para análisis estadísticos más complejos y avanzados. Su uso es poco común, pues la mayoría de las ocasiones al instalarse Excel no se tiene el cuidado de instalar todas las funciones dentro de las "herramientas" en forma completa y con ello la opción de "Análisis de datos", perdiendo la oportunidad de utilizar un medio muy poderoso para el análisis dentro de la estadística.

Por último debemos mencionar que ante el uso de un programa informático para el análisis estadístico, el que sea, debemos de realizar algunas consideraciones más allá del uso de herramienta propia en la educación.

Si bien, la computadora y los softwares estadísticos pueden y deben incluirse dentro de los programas de estadística como un instrumento de cálculo y representación gráfica, para analizar tanto los datos colectados por el estudiante mismo como aquellos proporcionados por el profesor; debemos preparar a los alumnos para recolectar, organizar, editar, almacenar, representar y analizar sistemas de datos con la computadora, cuya complejidad sea adecuada a su realidad.

Los objetivos del uso de la computadora comienzan por la comprensión de conceptos afines, empezando desde los básicos, como variables y conjunto de datos; no debemos olvidar el impacto que causa en los alumnos el empleo de sistemas de datos reales y significativos a su entorno: sus calificaciones escolares, los resultados de sus equipos deportivos favoritos, los noviazgos juveniles experimentados[9], etc.

Si la información analizada tiene significado y si para ellos es fiable, habrá mucho mayor disposición al momento de llevar el sistema de datos a la computadora y poder realizar sus análisis, eliminando un problema tradicional en la enseñanza de la estadística, el desfase entre la comprensión de los conceptos y los medios técnicos de cálculo para poder aplicarlos. [10]

También debemos considerar que la actual facilidad de empleo de procedimientos estadísticos, implica, el riesgo del uso no adecuado de estos. Es muy común que tras la recolección de un conjunto de datos se piense que es suficiente aplicar un programa estadístico para tener el análisis, pensando erróneamente que este automáticamente realizará la interpretación de los resultados obtenidos.

Debemos habilitar a los alumnos para planificar el análisis que desean desarrollar incluso antes de finalizar el diseño de su conjunto de datos, considerando que la culminación de este dependerá de ellos al momento de trasladar el valor numérico obtenido a la realidad de la cual fue extraído originalmente De otro modo, sus conclusiones pudieran carecer de valor y de riqueza explicativa. 


\section{Conclusiones}

A través de este documento sea han analizado algunos de los objetivos educativos que la disponibilidad de las computadoras y paquetes informáticos, plantea sobre la enseñanza de la estadística, reconociendo que:

Las TIC's ofrecen nuevas y poderosas herramientas de participación y toma de partido en forma deliberada en los procesos educativos.

El uso de la computadora ayuda a sostener el interés del estudiante para desarrollar tareas que son tediosas si se llevan a cabo de la manera tradicional, con lápiz y papel. estadístico.

También se han señalado las posibilidades didácticas de la computadora en el análisis

Se han evidenciado las "bondades" del uso de la hoja de cálculo de Excel como un "software" didáctico asequible en la enseñanza de la estadística.

No existen programas que por sí mismos resuelvan el problema de la enseñanza de la estadística . Es necesario un estudio concienzudo y profundo que permite el empleo de cualquier software estadístico en los programas educativos de estadística.

\section{Bibliografía}

BATANERO, C., ESTEPA, A. y GODINO, J. D. (1991). Análisis exploratorio de datos: Sus posibilidades en la enseñanza secundaria. Suma, 9, pp. 25-31.

BERENSON, Mark L. y LEVINE, David M. (1996). Estadística Básica en Administración. Edit. Prentice Hall. México.

BRENT, E. y MIRIELLI, E. (1991). Statistical navigator professional. Columbia, MO: The Idea Works, Inc.

GODINO, Juan D. (1995) . ¿Qué aportan los ordenadores a la enseñanza y aprendizaje de la estadística?. Versión revisada del artículo publicado en $U N O, 5$, pp.45-56

OROZCO, J. (2004) Uso Pedagógico de los programas Derive 6.1 y Cabri Geometry II plus, en las clases de Matemáticas. Proyecto de Innovaciones Tecnológicas en la enseñanza de las matemáticas y Ciencias. Colombia. http://www.scm.org.co/Subidos/855.Resumen.pdf

PÉREZ, César (2002). Estadística Aplicada a través de Excel. Edit. Prentice Hall. Madrid.

\section{Páginas de Internet.}

http://nctm.org/about/position_statements/position_statement_13.htm

http://www.eduteka.org/directorio/index.php?sid=271447174\&t=sub_pages\&cat=342

\section{Notas:}

[1] The Use of Technology in the Learning and Teaching of Mathematics; publicado por el Consejo Nacional de Profesores de Matemáticas. En: http://nctm.org/about/position_statements/position_statement_13.htm. Publicación de este documento en EDUTEKA: Febrero 07 de 2004. 
[2] (Octubre de 2003). Las declaraciones de la NCTM definen problemas particulares, hechos o necesidades y describen su pertinencia para la educación matemática. Cada declaración define la posición del Consejo en la materia y sugiere un curso de acción. La Junta Directiva de la NCTM aprueba todas las declaraciones. La fecha en paréntesis al final de cada declaración hace referencia a la fecha de aprobación.

[3] Proyectos publicados por EDUTEKA que usan la Hoja de Cálculo en varias materias http://www.eduteka.org/directorio/index.php?sid=271447174\&t=sub_pages\&cat=342 Publicación de este documento en EDUTEKA: Septiembre 20 de 2003.

[4] Pamela Lewis, Psicóloga de la Universidad de Sur África con un magíster en ciencias especializada en el área de computadores en educación. Profesora y coordinadora de informática en el Colegio "St. Luke", de Brookfield, Winsconsin, Estados Unidos.

[5] GODINO, Juan D. ¿Qué aportan los ordenadores a la enseñanza y aprendizaje de la estadística? pp.1

[6] GODINO, Juan D.¿Qué aportan los ordenadores a la enseñanza y aprendizaje de la estadística? pp. 2

[7] En: BERENSON y LEVINE, Estadística Básica en Administración, pp.7

[8] PÉREZ, César. Estadística Aplicada a través de Excel, pp. 2

[9] LÓPEZ, M., C. LAGUNES y S. HERRERA. Del Noviazgo a la Estadística...Un camino no tan largo. Acalán, en prensa.

[10] GODINO, Juan D. ¿Qué aportan los ordenadores a la enseñanza y aprendizaje de la estadística? pp.3.

(C) Ediciones Universidad de Salamanca 